\title{
DOSSIÊ
}

\section{Os Contornos e o Entorno da Nova Sociologia da Moral}

\section{Resumo}

Neste ensaio vou expor brevemente como vejo o desenvolvimento do campo da Sociologia da Moral, com foco em seu potencial fundamentalmente interdisciplinar, destacando os estudos e tradições que merecem ser incorporados à sociologia. A moral, como tema de investigação da ciência social, perpassa os campos da psicologia (social e do desenvolvimento), sociologia, antropologia, neurociências e economia. Aqueles entre nós implicados no seu desenvolvimento afirmam que ela serve de fundamento para toda a organização e interação social. Assumo, implicitamente, a posição do filósofo Charles Taylor e do sociólogo Christian Smith de que os seres humanos vivem envolvidos em teias de significados, pelas quais são moldados, conforme versões de "certo" e de "bem". Os seres humanos são fundamentalmente morais, não no sentido de serem convencionalmente altruístas ou de se preocuparem com os outros, mas de que as pessoas humanas, por serem seres sociais habitando um espaço social, devem assumir posições sobre temas relevantes nessas sociedades e grupos. As pessoas, de um modo geral, nesse meu paradigma, ancoram seus sentidos de si em posicionamentos morais, padrões que oferecem um solo a partir do qual dão sentido ao mundo através de lentes morais. Uma sociologia da moral compreende a formação dessas crenças, sua relativa imutabilidade ou as circunstâncias pelas quais elas mudam, sua influência sobre a ação e sua reconstrução retrospectiva diante de efeitos desajustados ou de pressões sociais.

Palavras-chave: Moral e interdisciplinaridade. Psicologia da moral. Antropologia da moral.

"Universidade de lowa (Estados Unidos) 


\title{
The Contours and Neighbors of the New Sociology of Morality
}

\begin{abstract}
In this essay, I will briefly cover the field as I see it developing, with an eye to its fundamentally interdisciplinary potential, highlighting studies and traditions that are worth incorporating into sociology. Morality as a topic of social science inquiry crosses the fields of psychology (developmental and social), sociology, anthropology, neuroscience, and economics, and those of us invested in its development argue that is serves as an underpinning for all social organization and interaction. Implicitly, I take the position that the philosopher Charles Taylor (1989) and the sociologist Christian Smith (2003) offer, that human beings are inextricably living within and shaped by webs of moral meanings, versions of the 'right' and the 'good'. Human beings are fundamentally moral, not in the sense of being conventionally altruistic or caring about others, but that human persons (SMITH, 2009) must, as a product of being social beings living in social space, take positions on issues important in those societies and groupings. People, as a rule, within my paradigm, anchor their senses of self within these moral positions, standards that offer an anchor from which to make sense of the world through a moral lens. A sociology of morality encompasses the formation of these beliefs, their relative immutability or the circumstances through which they change, their influence on action, and their retrospective reconstruction in the face of disconfirming feedback or social pressures.
\end{abstract}

Key words: Morality and interdisciplinarity. Moral psychology. Moral anthropology.

•Tradução: Carolina Fernandes 
Sociologias, Porto Alegre, ano 17, no 39, mai/ago 2015, p. 26-58

Introdução

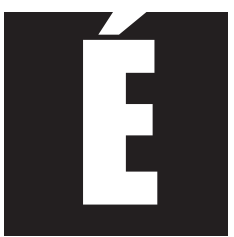

uma honra ser convidado para contribuir com o dossiê sobre Sociologia e Moral, junto e em homenagem a importantes membros da área. Neste artigo, abordarei brevemente a área, como a vejo se desenvolver, com um olhar para seu potencial fundamentalmente interdisciplinar, destacando estudos e tradições que merecem ser incorporadas à sociologia. A moral, como um tópico de investigação nas ciências sociais, envolve as áreas da psicologia (do desenvolvimento e social), sociologia, antropologia, neurociências e economia. Aqueles de nós dedicados ao seu desenvolvimento argumentam que ela serve de fundamento para toda a organização e interação social. Sob muitos aspectos, o campo da moral é o mais antigo na sociologia e, no entanto, esteve estagnado por muitos anos. Felizmente, parece ter sido revigorado em anos recentes e está maduro, com potencial para conectar subdisciplinas dentro da área, bem como as várias ciências sociais, de maneira geral.

Os escritos dos primórdios da sociologia tinham como foco questões sobre o espaço moral, crenças morais, compreensão moral, relações morais e ação moral na sociedade (HODGKISS, 2013). Tal interesse decresceu durante a segunda metade do século passado, talvez em resposta à natureza totalizadora percebida no projeto de Parsons para organizar e unificar a sociologia (JOAS; KNOBL, 2009) ou como reação à ideia de que estudar fenômenos "culturais", como crenças e valores, serviria para justificar desigualdades e opressão. De qualquer maneira, os últimos anos presenciaram um bem-vindo ressurgimento dos estudos sociológicos sobre a moral (HITLIN; VAISEY, 2010; HITLIN; VAISEY, 2013; LUKES, 2008; SMITH, 2003; TAVORY, 2011) com abordagens sobre antecedentes, processos e consequências da moral perpassando a disciplina (ABEND, 2014; 
BLAIR-LOY, 2010; FOURCADE; HEALY, 2007; IGNATOW, 2009a; STETS; CARTER, 2012; WINCHESTER, 2008) e com a introdução de uma nova seção sobre Altruísmo, Moral e Solidariedade Social na Associação Americana de Sociologia (JEFFRIES, 2014). Embora o estudo da moral como uma linha específica da sociologia seja relativamente novo, disciplinas correlatas desenvolveram trabalho sobre temas relacionados durante esse período de inércia sociológica. Psicologia social, antropologia, neurociência e psicologia do desenvolvimento têm explorado um grande número de micro e macro mecanismos compreendidos no domínio da moral, abrangendo desde crenças culturais (SHWEDER; MAHAPATRA; MILLER, 1987) até influências estruturais (INGLEHART; BAKER, 2000), comportamento grupal (HAIDT; HERSH, 2001), percepções individuais (GREENE, 2013).

De maneira geral, trato o estudo empírico da moralidade de modo pluralista e generalista. Seres humanos, colocando em termos banais, são complicados. Somos multifacetados, mutantes, em desenvolvimento, calculistas, emocionais, ilógicos e, muitas vezes, falta-nos autoconsciência. As influências sobre nosso comportamento abrangem desde processos históricos que não podemos identificar, até forças estruturais que limitam nossas escolhas, forças culturais que moldam nossa capacidade de pensar e sentir, padrões interacionais no âmbito das famílias e comunidades, experiências individuais peculiares e anomalias biológicas que moldam nosso comportamento e as percepções fundamentais de nós mesmos. Dada essa multiplicidade, o alcance das influências conscientes e inconscientes sobre nossas vidas - das quais a maioria das pessoas mal se apercebe -, considero um erro essencializar ou privilegiar qualquer disciplina, teoria ou paradigma para compreender a moral. Todas essas "peças móveis" (HITLIN, 2008) estão entrelaçadas, de modo complexo e, algumas vezes, inexplorado, com micro experiências sendo conectadas a macro fenômenos e macro forças incorporadas em micro interações (COLLINS, 1981; GIDDENS, 1984; TURNER, 1987). 
A sociologia, como disciplina, tenta estabelecer pontes entre as forças macro estruturais e culturais - que existem para além dos e anteriormente aos indivíduos que constituem a sociedade - e a experiência vivida e influências não percebidas sobre os comportamentos, pensamentos, sentimentos e percepções auto-reflexivas desses indivíduos. No âmbito da teoria sociológica, o final do século 20, envolveu debates sobre a natureza das conexões entre estrutura e ação, micro e macro, entre outros termos para esta relação recíproca. A moral é um caso especial e exemplar dessa relação recíproca. Construtos morais existem em múltiplos níveis analíticos. Unidades sociais, desde grupos até instituições e sociedades, comungam um sentido de moral. Na verdade, é isso que significa compartilhar uma "cultura", compartilhar significados e percepções morais, que formam os acordos tácitos do pertencimento a essa unidade social (VAISEY; LIZARDO, 2010). Isso está na essência do que Durkheim (1965[1912]) propôs como tema central para a sociologia. Contudo, existe simultaneamente a "ordem da interação" (GOFFMAN, 1983), uma ordem social fundamentalmente moral (RAWLS, 1987; 1989; 2010), regras comportamentais estabelecidas nas interções face-a-face que permeiam os agrupamentos sociais. As instituições canalizam orientações coletivas, voltadas para o justo e o bom (JACKALL, 1988; SNYDER, 2013), enquanto atores sociais individuais desenvolvem uma compreensão socialmente moldada de si mesmos como pessoas "morais" (HITLIN, 2008; SMITH, 2003; STETS; CARTER, 2006).

Este artigo inclui um panorama certamente breve do campo da sociologia da moral e de algumas importantes disciplinas correlatas, uma vez que essas podem aportar conhecimento à nossa disciplina. Assumo, sem reservas, a posição do filósofo Charles Taylor (1989) e do sociólogo Christian Smith (2003), de que os seres humanos estão vivendo inextricavelmente dentro e são moldados por redes de significados morais, versões do "certo" e do "bom". Os seres humanos são fundamentalmente morais, 
não no sentido de serem convencionalmente altruístas ou de importarem-se com os outros, mas de que pessoas humanas (SMITH, 2009) devem - em razão de serem seres sociais vivendo num espaço social - assumir posições sobre questões importantes naquelas sociedades e grupos. Agir de maneira neutra ou sistematicamente não engajar-se em debates e discussões sobre questões morais localmente importantes seria o mesmo que ser visto como alheio à ordem local, de certa forma, ter colocada em dúvida sua capacidade intelectual. Ser um ator estritamente racional é evidenciar formas de autismo social; seres humanos devem demonstrar, ao menos, um mínimo de preocupação com as crenças de outros, ou correm o risco de ser julgados como membros não adequados para aquela comunidade social (HITLIN; ANDERSSON, 2013). Tampouco um membro ativo, integrado à comunidade social, pode adotar posições morais arbitrárias ou mudar constantemente suas crenças fundamentais. Não somos seres perfeitamente coerentes, mas, na modernidade, alegar que não temos um núcleo moral é visto como uma falha fundamental de caráter. Crenças externas sobre certo e errado, obrigações e proibições morais (BANDURA, 2008) são internalizadas pelos indivíduos (FIRAT; MCPHERSON, 2010) e orientam o julgamento de ações - próprias e de outros - ou restringem aqueles pensamentos e comportamentos vistos como imorais, errados ou imperdoáveis - algo que em outros textos eu denomino "luzes brilhantes" ou "linhas brilhantes" (HITLIN, 2008).

Isso não significa que seres morais, ideologias morais ou sistemas morais são tão coerentes e indiferenciados quanto os trabalhos de Parsons ou Durkheim podem sugerir. Vivemos em um mundo de estruturas, códigos e ideologias concorrentes, onde não importa qual seja o seu sistema de crenças, você está ciente dos sistemas concorrentes. Isso pode levar as pessoas a acreditarem mais, ou menos, em suas próprias ideologias; os efeitos de sistemas morais plurais sobre o indivíduo são questões empíricas, com as influências provavelmente divergindo através do tempo e espaço. 
Diferentes grupos, países ou subgrupos dispõem de diferentes referências para decidir o certo e o errado, e algumas dessas abordagens são incomensuráveis. Contudo, os valores (SCHWARTZ, 2012) mais importantes para um grupo ou uma pessoa são, para esses, incontestáveis e vistos como evidentemente verdadeiros (JOAS, 2000). Em meu paradigma, as pessoas, via de regra, ancoram seu senso de si nessas posições morais, padrões que oferecem um fundamento a partir do qual dar sentido ao mundo através de lentes morais. Uma sociologia da moral abrange a formação dessas crenças, sua relativa imutabilidade ou as circunstâncias em que elas mudam, sua influência sobre a ação e sua reconstrução retrospectiva frente a respostas invalidadoras ou pressões sociais. No entanto, vivemos em uma sociedade pluralista, muito mais alinhada à noção de Weber, da complexidade da vida social moderna (HITLIN; VAISEY, 2013) e, portanto, supor um sistema puro ou totalista é, provavelmente, uma tarefa inútil.

\section{Definindo Moralidade}

Desde o surgimento das ciências sociais, a moralidade foi considerada essencial para as relações humanas. Nos primeiros trabalhos de Durkheim (LUKES, 1985) e para Adam Smith (JAHODA, 2007) os termos "social" e "moral" eram considerados intercambiáveis. Durkheim mudou para o termo "moral" para adequar-se a atuais tendências na linguagem, mas preferia o mesmo o termo social. Os fundadores da sociologia interessavam-se pelas dimensões morais da sociedade (LEVINE, 2010; POWELL, 2010). A sociologia da moral, latente por longo período durante o século 20, ressurgiu como campo de estudo apenas no início do século 211․ Em suas relevantes conclusões, Abend (2010) e Lukes (2010) ofere-

1Para uma síntese dos temas desta tendência, ver Abend (2008). 
cem algumas críticas construtivas às questões específicas envolvidas na abordagem sociológica. Eles se concentram em questões tais como: em que medida os sociólogos deveriam adotar uma perspectiva moral particular e que é preciso reconhecer que este é um tópico de estudo no qual seres humanos podem achar difícil libertarem-se de suas próprias propensões (LUKES, 2008).

O estudo da moralidade é fundamentalmente interdisciplinar. A nova seção da Associação Sociológica Americana denominada Altruísmo, Moral e Solidariedade Social aponta para um dos principais usos do termo "moral" na sociologia, aquele das forças coesivas, pró-sociais subjacentes à sociedade. Meu uso de moral baseia-se nessa noção - certamente, noções convencionais de comportamento adequado ou recomendável estão na base daquilo que estudamos - mas sugiro um entendimento mais amplo. A moral inclui o estudo do altruísmo, uma variedade específica de orientações e comportamentos morais que visam promover o bem-estar dos outros (PILIAVIN, 2008), bem como conceitos de solidariedade social, as forças que conduzem grupos e instituições sociais à coesão. A concepção sociológica de moral envolve dois sentidos (HITLIN; VAISEY, 2013): primeiro, existem os padrões antes citados, supostamente universais, de certo e errado, os quais se vinculam a questões de equidade, justiça e dano (TURIEL, 2002). Nesse sentido, "moral" é, de um modo geral, equivalente a "pró-social" ou "bom" e seu oposto é "imoral". Essa abordagem domina boa parte da psicologia moral e nichos da sociologia, especialmente no estudo do ego moral (STETS; CARTER, 2012) e do altruísmo.

Um segundo sentido envolve as próprias interpretações, e foca mais no conteúdo local de expectativas morais do que em designá-las a priori. Esse uso de moral extrapola o sentido de ajudar ou provocar dano a outros, envolvendo questões que Abend (2011) denomina entendimentos "densos"; temas como piedade, dignidade, exploração e fanatismo. Essa 
Sociologias, Porto Alegre, ano 17, no 39, mai/ago 2015, p. 26-58

forma de moralidade vê o conteúdo de sistemas morais mais com uma variável que é influenciada por uma série de fatores estruturais, culturais e históricos. É uma perspectiva que faz do conteúdo da moral a problemática empírica e teórica em si. O que as pessoas nessa instituição/ sociedade/grupo consideram moral, e o que consideram inaceitável? Este é um conjunto mais rico de potenciais áreas de estudo e seu oposto não é a "imoralidade", mas, simplesmente, algo que é "não-moral", fora do que é considerado moralmente relevante naquela circunstância. Abend (2014) usa a história da formação em Administração para demonstrar a importância de outro sentido ainda - o de "pano de fundo moral" - por meio do qual os sociólogos podem estudar as pré-condições que moldam os pressupostos que dão sentido a debates morais específicos.

Em sua acepção sociológica, a moral cobre tanto os sentidos tácitos como os explícitos compartilhados, que formam o clássico tema sociológico de "normas e valores". As sociedades humanas estabelecem padrões para julgar seus membros quanto às formas apropriadas, recomendáveis e indignas de pensar, sentir e agir. Isso significa que qualquer pensamento ou ação está potencialmente sujeito à aprovação ou louvor moral, mas é também passível de ser visto como violação da ordem moral. Qualquer ato pode identificar seu iniciador como pessoa, empregado ou membro de grupo de "má índole" e esses entendimentos são transmitidos a novos membros, podendo ser objetos de discussão ou disputa entre membros de grupos, tais como crianças discutindo com seus pais, ou partidos políticos debatendo suas prioridades em uma sociedade específica.

Podemos examinar a moral como uma variável independente, explorando as ramificações de conteúdo moral específico em relacionamentos, ações ou distribuição de bens e serviços em qualquer população. Uma perspectiva sociológica sobre esses, frequentemente, aponta para paradigmas mais ecologicamente válidos do que os paradigmas psico- 
Sociologias, Porto Alegre, ano 17, no 39, mai/ago 2015, p. 26-58

lógicos tradicionais - frequentemente experimentais (HITLIN; VAISEY, 2013). Definir o conteúdo também permite a investigação dos antecedentes sociais estruturais dos vários aspectos morais da vida social, como o interesse de Schwalbe (1991) em ligar o indivíduo moral à sociedade ou a análise de Starks e Robinson (2007) de como a religião molda as perspectivas morais.

\section{Abordagens sociológicas para um tema multifacetado}

A sociologia, como disciplina, obtém força - e algumas críticas - pela grande variedade de abordagens teóricas e empíricas sob nosso domínio. Não temos a coesão metodológica disciplinar da psicologia, por exemplo, ou tampouco o paradigma teórico da economia (apesar desse estar se ampliando lentamente). A pesquisa sociológica varia entre qualitativa e quantitativa, micro e macro e abrange todas as facetas da vida humana política, familiar, econômica e assim por diante. Isso significa que resumir o estado do conhecimento sobre um tema como a moral - que, pelas definições fornecidas aqui, navega por todas as dimensões da vida social organizada - é uma tarefa hercúlea e destinada a ser parcial e incompleta. Dito isso, forneço aqui um breve, e reconheço, limitado mapa de algumas tentativas notáveis de entender a moral a partir de perspectivas teóricas e empíricas. Esses projetos deveriam formar o corpus do que venha a tornar-se este incipiente campo que ressurge.

Talvez o apelo mais forte para que, na última década pelo menos, a moral estivesse mais no centro da pesquisa sociológica venha de um pequeno livro de Christian Smith (2003), seguindo as pegadas teóricas de Charles Taylor $(1989)^{2}$. O livro defende a centralidade da moral para com-

$\overline{{ }^{2} \text { Ver também Calhoun (1991) }}$

http://dx.doi.org/10.1590/15174522-017003902 
Sociologias, Porto Alegre, ano 17, no 39, mai/ago 2015, p. 26-58

preender os indivíduos e a cultura que os molda. Muitas outras abordagens notáveis sobre o tema surgiram nesse período, incluindo Joas (2000) e seu foco na natureza pragmática de descobrir valores pessoais, Lukes (2008) focando em questões de relativismo moral e de como essas se refletem em estudos científicos sociais da moralidade, e Sayer $(2005 ; 2011)$ que vincula classe social com questões de percepção moral e defende noções de moral cautelosamente prescritivas nas ciências sociais. Alguns teóricos mais jovens (ABEND, 2011; 2012; TAVORY, 2011) também ofereceram críticas e correções valiosas sobre como este campo incipiente deveria se desenvolver. Abend, em particular, delineia os contornos e a importância do "pano de fundo moral", das noções tácitas, frequentemente implícitas, socialmente compartilhadas que estabelecem as precondições para o tipo de temas geralmente estudados sob a umbrela da moral (ABEND, 2014). Este trabalho se baseia em abordagens clássicas das dimensões morais da cultura que moldaram o subcampo e refinaram-se no estudo mais amplo do fenômeno moral (LAMONT, 1992; WUTHNOW, 1992).

Uma clara vantagem da investigação sociológica é seu foco na natureza situada da interação, o que os indivíduos desenvolvem anteriormente e como resultado dessas interações. Muito do esforço performativo que ocorre na autoapresentação interativa de qualquer pessoa (GARFINKEL, 1967; GOFFMAN, 1959) e o tipo dessa interação (GOFFMAN, 1983) estão repletos de significados morais e de potenciais sanções morais para violações da ordem normativa ${ }^{3}$. As pessoas presumem que os outros possuem uma "essência" (KATZ, 1975) e esses são alvo de escrutínio moral, principalmente quando seu comportamento deixa de atender aos padrões sociais ou acordos previamente estabelecidos. Mais recentemente, Stets e Carter (2012)

3Para uma discussão dessas questões e de como os indivíduos desenvolvem uma percepção de si mesmos como seres morais, ver Hitlin (2008). 
detalharam alguns dos mecanismos psicológico-sociais, através dos quais significados situados convergem com crenças pessoais fundamentais para motivar e dar sustentação à identidade moral de um indivíduo. A moral é subjacente às ações que em geral impedem comportamentos anormais e atos criminosos (ANTONACCIO; TITTLE, 2008; WIKSTROM et al., 2010; WIKSTROM, 2007).

A investigação sociológica macro orientada remonta às origens do campo no que se refere à teorização sobre o modo como fatores como classe social moldam as perspectivas morais, e inclui as obras de Marx e Engels, ou de teóricos como Weber (influenciado por Nietzsche), sobre como percepções individuais da realidade moral são formadas de maneira distal pela organização dos meios de produção, e como os grupos no poder podem ser exitosos na propagação de cosmovisões morais que definem o mundo em seu benefício. As perspectivas morais são influenciadas por uma variedade de fatores macro (BLACK, 2011), desde histórias culturais (INGLEHART; BAKER, 2000) até tradições religiosas (BADER; FINKE, 2010). Em uma sociedade específica, os significados morais também podem mudar através dos tempos (ZELIZER, 2007; 1979), com diferentes lógicas morais entrelaçando-se de forma complexa, através de domínios como família e mercado. Uma importante conclusão dessa obra é que significados morais são parte de um espaço compartilhado e não simplesmente algo localizado nas mentes dos seres humanos. Organizações, por exemplo, podem moldar a maneira como as pessoas vinculam valor moral a certos atos, tais como doação de órgãos (HEALY, 2006).

Talvez, o mais popular campo em desenvolvimento da sociologia seja o do estudo da cultura, o qual tem se revelado uma área fértil para envolver questões morais. Alguns dos trabalhos mais importantes vêm da obra de Stephen Vaisey (2007), que se concentra na forma como a moralidade define a ordem moral coletiva, e de sua adoção de um duplo processo psicoló- 
gico funcional, para demonstrar como o conhecimento de padrões morais que só estão disponíveis implicitamente - não são fáceis de ser articulados - pode prever comportamentos morais subsequentes (VAISEY, 2009). Ignatow (2009a; 2009b) e Winchester (2008) oferecem importantes reparos culturais aos, com frequência excessivamente cognitivos, estudos sobre a moral - conforme são encontrados em abordagens psicológicas clássicas que têm sido ampla e inquestionavelmente incorporadas às discussões sociológicas da moralidade -, demonstrando a natureza internalizada e não-articulada das crenças e práticas morais.

As abordagens sociológicas tendem a se concentrar nos substratos sociais/morais subjacentes que informam as análises encontradas em modelos econômicos de troca, e encontram mais poder incorporado nas instituições e na cultura do que simples racionalidade na atribuição de sentido a essas trocas. Fourcade e Healy (2007) ilustram maneiras em que noções oriundas do sistema econômico foram inculcadas nas noções de moral. Dois economistas, críticos de sua própria disciplina, Halteman e Noell (2012), insinuam o quão importantes podem ser as influências sociais, interpretando o modelo do ator associal racional como um tanto problemático. Fora da disciplina, mas demonstrando de maneira importante a natureza das crenças culturais para estruturar intercâmbios situados, Henrich e colegas (HENRICH; HEINE; NORENZAYAN, 2010; HENRICH; HEINE, 2010) sugerem que o tamanho relativo do contexto interacional local de um indivíduo, afeta os julgamentos no nível individual - em contraste com aquelas abordagens que consideram que os seres humanos apresentam certas reações morais inatas frente a trocas desiguais. Em Scott (1976) encontramos uma clássica demonstração de como a situação de desvantagem extrema de uma população camponesa molda uma ordem moral local específica, mais fundada em direitos e justiça social do que num foco econômico em bens materiais. 
Esses substratos existem em diversas formas de organização humana, as quais se estruturam com base em diferentes recursos e poderes. Tais organizações variam desde as formais, como empresas com níveis gerenciais e burocracia (JACKALL, 1988) até meios mais informais pelos quais o estado incute questões morais nas suas políticas enunciadas (STEENSLAND, 2010). Em ambos os casos, os indivíduos recebem fortes sinais de entidades mais poderosas, que de diferentes formas controlam suas vidas, sobre quais prioridades são "justas" e "morais". Como mostra Jackall, muitos gerentes intermediários em corporações norte-americanas mudam seu senso de certo e errado, assumindo aqueles de seus supervisores diretos e deixando as questões morais convencionais fora do ambiente de trabalho. A moralidade configura o modo como as pessoas conciliam potenciais conflitos em suas vidas - entre casa e trabalho, por exemplo (BLAIR-LOY, 2003; 2010) -, e as prioridades estabelecidas por elas nos múltiplos domínios da vida humana moderna (ELDER, 1994). O trabalho de Healy (2006) já mencionado revela o poder das instituições na padronização das decisões individuais sobre doação de órgãos, demonstrando como o mais individual dos atos é altamente influenciado pelos significados inerentes a organizações sociais mais abrangentes.

Finalmente, uma análise sociopsicológica da moralidade dentro de um marco sociológico centra-se em ambas as situações - circunscritas a significados e expectativas morais que constituem a ordem da interação - e nas cosmovisões morais de indivíduos que entram, sancionam e deixam aquelas situações. Existe uma diversidade de construtos, extensamente pesquisados, que podem informar a análise sociológica de códigos morais situados e internalizados, tais como emoções morais (TURNER; STETS, 2006a; 2006b), confiança (USLANER, 2002) e justiça (HEGTVEDT; SCHEURMAN, 2010). Esse trabalho oferece abordagens sociológicas, mas é fundamentalmente interdisciplinar. 
Sociologias, Porto Alegre, ano 17, no 39, mai/ago 2015, p. 26-58

\section{Disciplinas Correlatas e Potenciais Contribuições Sociológicas}

Psicologia. O renascimento sociológico do tópico acompanha os passos de um crescente foco sobre a moral na psicologia (GRAHAM et al., 2011; HAIDT, 2001; HAIDT; KESEBIR, 2010; LAPSLEY; NARVAEZ, 2004; SKITKA; BAUMAN; MULLEN, 2008; SUNAR, 2009). Durante o período em que a sociologia esteve menos dedicada a questões de valores e moralidade (SPATES, 1983), o trabalho da psicologia foi consideravelmente influente. Kohlberg (1981) desenvolveu uma célebre tipologia do desenvolvimento moral, que teve grande impacto e foi também alvo de forte crítica feminista (GILLIGAN, 1982). Esse debate específico tem suporte empírico mínimo no que concerne a explicar diferenças de gênero (JAFFEE; HYDE; 2000), mas o esforço de sistematização do juízo e funcionamento morais foi interpretado de modo amplo, conduzindo a tentativas influentes de ancorar a psicologia moral em alguns temas centrais, especialmente justiça e equidade (TURIEL, 1983; TURIEL; KILLEN; HELWIG, 1987). A moral tornou-se central para teorias que tentam explicar o eu e a cognição humana (BANDURA, 1999; 2004; BANDURA et al., 1996).

Esse esforço de centralizar o estudo da moral em um ou dois temas tem seu atrativo - ou seja, parcimônia científica - mas um trabalho mais recente ampliou essas ideias para explorar uma gama mais vasta de potenciais fundamentos morais, ultrapassando o foco na justiça para abranger pureza, autoridade e lealdade como outros objetivos morais humanos naturalmente desenvolvidos (GRAHAM et al. 2012; HAIDT; JOSEPH, 2008). Atualmente, os trabalhos estão focados em encontrar o equilíbrio entre pensamento e sentimento morais (FRIMER; WALKER, 2008) ou em tentar medir adequadamente a noção pró-social de identidade moral (AQUINO; REED, 2002; REED; AQUINO, 2003). 
Sociologias, Porto Alegre, ano 17, no 39, mai/ago 2015, p. 26-58

A psicologia da moral se segmenta em função daquilo que os teóricos sugerem como fundamentos da moral - fatores cognitivos versus fatores emocionais. O trabalho clássico, mencionado acima, é consideravelmente orientado para a cognição, mas o teor geral da pesquisa nas duas últimas décadas dificulta o foco exclusivo na cognição como se esta estivesse divorciada da emoção. O trabalho do neurologista Damasio (1999; 2003) tem sido um propulsor em demonstrar como são importantes as emoções, até mesmo para o processo supostamente não-emocional de ser lógico. Diversas decisões potenciais contêm "marcadores somáticos", nuances emocionais que dão o tom e ajudam a orientar até mesmo a seleção racional de alternativas. Em outras palavras, não existe um pensamento de caráter social sem marcadores emocionais ${ }^{4}$; indivíduos com um déficit neste tipo de pensamento são frequentemente vistos como marginais quanto a seus modos de interação. Isso conduz a um crescente foco na natureza do ego cognitivo/emocional em sua interseção com ações e escolhas morais (BLASI, 2004; WALKER, 2000), incluindo um reconhecimento da ambiguidade presente no mundo interacional. Nessa perspectiva, tem crescido o número de pesquisas sobre o tema das emoções morais (HAIDT, 2003) incluindo algumas importantes emoções interacionais como empatia (HOFFMAN, 2000) e vergonha (TANGNEY; STUEWIG; MASHEK, 2007), das quais a última foi incorporada aos modelos sociológicos do ego e da sociedade (SCHEFF, 2000; 2003).

Uma área em que a psicologia certamente ultrapassou a sociologia é a da conceituação e medição do desenvolvimento da moralidade, especialmente no que tange às primeiras etapas da vida humana. Psicólogos do desenvolvimento há tempo mostram interesse nos aspectos da primeira infância que levam ao desenvolvimento da moral, com alguns

${ }^{4}$ Ver também Prinz (2007)

http://dx.doi.org/10.1590/15174522-017003902 
pesquisadores (por ex., KOCHANSKA, 2002; KOCHANSKA et al., 2004) sugerindo que o temperamento da primeira infância provoca diferentes padrões de desenvolvimento da consciência. Crianças compreendem princípios abstratos mais completamente à medida que crescem, embora sejam em geral moralmente motivadas, ainda que de maneira diferente nos primeiros anos (NUNNER-WINKLER, 1998). O desenvolvimento da moral ocupa um capítulo importante em uma das etapas mais importantes do desenvolvimento das crianças (KAGAN, 1994[1984]) e pesquisas recentes sobre a adolescência também destacam a importância de se desenvolver um senso de moral nos adolescentes (HART; CARLO, 2005).

Neurociência e Biologia. Sociólogos têm, muitas vezes, evitado o foco direto na biologia, mas na medida em que as ciências se tornam cada vez mais interdisciplinares, colocamos-nos em desvantagem como campo se falharmos em envolver corretamente tais áreas (FREESE, 2008; MASSEY, 2002). Neurologia é uma arena especialmente importante para um diálogo com a sociologia (FRANKS, 2010), em vista dos elementos fundamentais do trabalho de George Herbert Mead (MEAD, 1932; 1934) e do que era, na época, uma vaga conceituação da mente, que pode agora ser mais completamente examinada (FIRAT; HITLIN, 2012). Muitas teorias sociológicas do ator são baseadas em hipóteses cognitivas que hoje são mais passíveis de ser testadas do que em épocas anteriores. Além disso, melhoramos as ferramentas teóricas e empíricas para estudar a miríade de caminhos através dos quais a cultura torna-se internalizada nos níveis mental e corporal (FIRAT; MCPHERSON, 2010).

Alguns dos mais importantes trabalhos antes mencionados vêm da obra de Damasio (2005), que evidencia a base emocional do julgamento moral. As próprias emoções são, em boa parte, culturalmente formadas e canalizadas (TURNER; STETS, 2006b). Sendo assim, confiar nas emoções não representa, de modo algum, ameaça à primazia do social na 
compreensão do comportamento; na verdade, ela complementa campos sociológicos consagrados. Conforme apontam Damasio e sua equipe, memorizamos informações sociais complexas, as quais são disparadas quando enfrentamos dilemas morais, sensações que nos conduzem a soluções adaptadas a nossas experiências e nossa cultura. Greene e Haidt (2002) operam neste campo, sugerindo que um sistema psicológico humano dual (um rápido, instintivo, e um lento, o raciocínio consciente) está implicado no julgamento e ação morais. Simplificando, humanos não agem de maneira tão lógica quanto às teorias clássicas de Kohlberg sugeriam. Ao contrário, nos comportamos de modo mais intuitivo, apenas "sabendo" o que parece ser a coisa moralmente certa a fazer (em nossa cultura) e, depois, usando um raciocínio post hoc para descobrir formas de justificar esta intuição. Esse trabalho se consolidou na psicologia moral (SINNOTT-ARMSTRONG, 2008).

Seres humanos são, basicamente, animais - e recentes pesquisas evolucionárias situam nossa capacidade moral na evolução das espécies. Este processo é amplamente compreendido como sendo fundamentalmente social, não a simples sobrevivência dos mais fortes, que parece privilegiar o comportamento individual em detrimento daquele do grupo. De Waal (2006; 2009; FLACK; DE WAAL, 2000) resume a literatura que trata de como a evolução, em contextos grupais, prediz o comportamento moral humano atual e seu e funcionamento, evidenciando diversas similaridades desenvolvidas que ajudam a compreender a importância de significados morais compartilhados para a vida como uma espécie social (ver também KREBS, 2011). Na sociologia, Jonathan Turner (MARYANSKI; TURNER, 1992; TURNER, 2010), há muito, tem ilustrado a natureza fundamentalmente social da evolução da espécie humana e os limites biológicos que ela coloca sobre o funcionamento nas sociedades modernas (ver também MASSEY, 2002). 
Antropologia. A história dos fundamentos morais segue uma ordem interessante, com Kohlberg sugerindo um tipo de sistema moral, Gilligan dois e, depois, Haidt e sua equipe, cinco ou seis (eles ainda estão refinando suas medidas). Curiosamente, antropólogos sugeriram tipologias de três ou quatro, respectivamente, apenas parcialmente introduzidas na sociologia, mas que são muito promissoras para investigações futuras e fundamentalmente vinculadas a questões de cultura e estrutura, que uma sociologia da moralidade consideraria atraente.

Richard Shweder discutiu os limites da abordagem de Kohlberg, sugerindo que o marco da justiça é apenas um entre três marcos utilizados pelas culturas para estabelecer um senso coletivo de moral (SHWEDER; MAHAPATRA; MILLER, 1987; 1990). Shweder e seus colegas classificaram a abordagem de Kohlberg como o paradigma de uma "ética da autonomia", uma visão de mundo racional que se centra na justiça e no dano como moedas de julgamento moral. A essa, ele acrescenta duas outras potenciais éticas culturais: a "ética da comunidade" e a "ética da divindade". Essas duas éticas representam sistemas morais mais orientados à comunidade, menos focados no bem individual e mais no coletivo. Esse foi referenciado como o modelo dos Três Grandes e discute a centralidade da experiência emocional como uma lente através da qual a moral é demonstrada e experimentada por membros de uma cultura. Alguns acadêmicos (ROZIN et al., 1999) vincularam emoções morais específicas ao fato de serem mais ou menos predominantes em sociedades com aquela ética moral respectiva. As pessoas sentem raiva por violações da autonomia, desprezo por violações da comunidade e repulsa por violações da divindade.

Fiske assume uma abordagem mais orientada à estrutura, para estabelecer potenciais bases morais (FISKE, 1992; FISKE; HASLAM, 2005). Ele propõe quatro formas básicas de relações sociais, que estimulam expectativas e comportamentos previsíveis. Esses modelos de Regulação 
Sociologias, Porto Alegre, ano 17, no 39, mai/ago 2015, p. 26-58

das Relações (RR) pretendem fornecer algumas bases sistemáticas para as várias formas de comprometimento moral experimentado e a capacidade de prever quais formas de relarelação levarão as pessoas a recorrer a um desses quatro fundamentos morais, ao invés de outro qualquer (RAI; FISKE, 2011). Essas quatro relações humanas potenciais são: a) hierarquia (escala de autoridade); b) unidade (partilha comunitária); c) igualdade (correspondência) e d) proporcionalidade (valor de mercado). Cada tipo de estrutura suscita motivações morais específicas, e uma relação que apela para uma forma de moralidade (digamos, valor de mercado) invoca lógicas morais específicas. Isso significa que indivíduos que aceitam aquela forma de relacionamento irão vivenciar de forma muito negativa uma violação da mesma. Sociedades humanas primitivas orientavam-se por escalas de unidade e de autoridade, enquanto as formas mais recentes de organização social priorizam a igualdade e a lógica de mercado. Essa, também, é uma teoria que privilegia o papel das emoções, sinais que, na maioria das vezes, surgem como reações a violações das expectativas culturais (por exemplo, raiva e repulsa) ou como relacionamentos construtivos (por exemplo, amor).

\section{Considerações Finais: que rumo o campo deve seguir?}

Esta é uma época promissora para esse novo antigo subcampo sociológico. Dada a prevalência de questões morais como base para a solidariedade cultural, o tema nunca esteve totalmente fora da discussão sociológica, mas a diminuição de sua importância parece estar se revertendo agora (HITLIN; VAISEY, 2013). Vários projetos e setores organizacionais estão oferecendo a possibilidade de estabelecimento de redes, trabalho interdisciplinar e auto-identificação para aqueles membros da nossa disciplina cujo trabalho tem relação com essas questões 
centrais. Em vista da perspectiva que utilizo para identificar o fenômeno moral, poder-se-ia dizer que o campo tem estado preocupado com essas questões desde o princípio. Nenhum grande teórico pode discutir significado mutuamente inteligível, universos simbólicos compartilhados ou ação social mutuamente constituída sem fazer referência às várias formas emocionais e simbólicas de significado intersubjetivo que ligam grupos e seus membros. Mas dizer que a moralidade está em todos os lugares é o mesmo que dizer que em nenhum lugar ela é significativa e, certamente, muitos comportamentos habituais ficam fora do domínio daquilo a que nós geralmente nos referimos como o domínio da moral. Contudo, até mesmo aqui, existe um "pano de fundo moral" (ABEND, 2014) que torna inteligíveis e aceitáveis as trocas do cotidiano e os sociólogos precisam estar cientes dessas influências distais e, até mesmo, definicionais, sobre as formas de ação e organização que constituem boa parte do campo atual.

Meu entendimento geral do campo, contudo, diz que podemos ver questões morais tanto como constitutivas de sociedades (Weber), quanto como indicadoras de suas formas sociais (Durkheim), como forças de privilégio e opressão (Marx) e como variáveis dependentes, moldadas por questões de classe, gênero e nacionalidade. Questões e preocupações morais abrangem desde definições sociais daqueles que "merecem" auxílio do governo, até o senso de decência gerado localmente, que pode ser violado na rua. Podemos olhar as questões morais como englobando as diferenças essenciais entre sociedades e na interação entre definições legais e sociais de certo e errado, bem como os movimentos sociais que ajudam a exemplificar determinadas práticas como aceitáveis ou tabus. Indivíduos são definidos - como eu detalho melhor em outros trabalhos (HITLIN, 2003; 2008) - por suas prioridades morais autojustificadas. Consideramos as pessoas de nossos prórpios grupos como sendo mais morais do que aquelas que definimos como as "outras"; vemos nossas próprias 
ações - devido a uma série de vieses cognitivos - como aceitáveis, até mesmo quando as condenamos em outros. E somos mais generosos com nossos próprios sentimentos e ações, uma vez que as pessoas geralmente estão convencidas de sua própria decência moral, quando não consideram a si mesmas como exemplos de moralidade (GIGERENZER, 2010). Mais do que um modelo funcionalista totalizador de sociedade, eu (e muitos outros profissionais atualmente) adoto a visão Weberiana de uma natureza multifacetada da moral na sociedade, bem como a de que questões morais são pontos privilegiados para contestação entre grupos e nações.

Ainda resta muito trabalho a ser feito, por exemplo, no campo das classes sociais e experiências morais. Lamont (1992) detalha diferenças sistemáticas entre as sociedades francesa e americana, enquanto Sayer (2005) detalha a forma como as preferências das classes presumidamente mais altas servem para reproduzir sistemas de estratificação social. Na psicologia, descobrimos que, em relação a emoções morais, diferenças de classe são melhores preditores de juízos do que diferenças culturais (HAlDT; KOLLER; DIAS, 1993). Parece claro que a classe social molda a experiência moral e, possivelmente, que essa experiência reproduz a estrutura de classes, mas esse é um campo maduro para investigações futuras.

Há necessidade de pesquisas com maior enfoque intercultural sobre as relações de uma série de fatores sociológicos com diferentes ações, comportamentos, sentimentos e bases morais. Temas como religião, regimes políticos, história cultural e recursos moldam as sociedades em muitas formas, algumas delas previsíveis, algumas delas conectadas a histórias culturais particularistas (INGLEHART; BAKER, 2000). Meu mapeamento do campo está, em grande parte, centrado na América do Norte e o inglês permanece o idioma corrente em boa parte da sociologia. Certamente precisamos valorizar - e mesmo estimular - comparações, colaboração e abertura para formas alternativas de interpretação, estruturação e análise 
entre nações. Deveríamos usar uma vasta gama de recursos e perspectivas para explorar as formas de moralidade social, situacional, grupal e individual que fundamentam a ordem social.

Devemos ter cuidado para não presumir que todos os exemplos de moralidade são bons ou que pessoas são sempre louváveis quando agem de maneira moral. Argumentos e justificações morais têm sido explorados por todos os tipos de atos horrendos (OSOFSKY; BANDURA; ZIMBARDO, 2005; STAUB, 2003). Estudar a moralidade não é apenas estudar os nobres esforços da humanidade. É focar naquelas forças que unem os grupos, independentemente de nosso julgamento moral sobre os objetivos daqueles grupos. Como antes, adoto também a distinção weberiana entre análise e defesa (advocacy). Em nosso trabalho formal como cientistas sociais, precisamos ser cautelosos ao alegar que oferecemos uma interpretação especial sobre o que "deveria" ser feito em relação à sociedade e seus membros. Podemos tentar elucidar a satisfação e a relação entre os vários atores que formam os sistemas sociais; sou cauteloso em afirmar que a sociologia seja o centro a que as pessoas devem se voltar para orientação moral. Sou um pluralista cultural, não um absoluto relativista, mas o próprio conhecimento de fundamentos morais alternativos, em si, deveria nos tornar mais modestos em fazer afirmações morais universalistas (SHWEDER; HAIDT, 1993). No entanto, parece haver, de fato, alguns padrões gerais na organização social, na hierarquia e no papel das experiências humanas constitutivas da moralidade em sustentar a ordem social. O desafio para o campo é, a partir das importantes correntes intelectuais, presentes desde o início da disciplina, evoluir tanto com teorizações originais quanto com melhores técnicas empíricas. Este é um momento oportuno para atuar nesse campo e espero que esta publicação especial possa ser uma pequena contribuição para conferir relevância ao mesmo na disciplina. 
Sociologias, Porto Alegre, ano 17, no 39, mai/ago 2015, p. 26-58

Steven Hitlin - Ph.D Departamento de Sociologia Universidade de Wisconsin, professor Adjunto e diretor de Pós-Graduação da Universidade de lowa., e membro da seção Altruísmo, Moralidade e Solidariedade Social da Associação Americana de Sociologia. $>$ steven-hitlin@uiowa.edu

\section{Referências}

1. ABEND, Gabriel. Two Main Problems in the Sociology of Morality. In: Theory and Society 37(2), 2008, p. 87-125.

2. ABEND, Gabriel. What's new and what's old about the new sociology of morality. In: HITLIN, Steven; VAISEY, Stephen (ed.). Handbook of the Sociology of Morality. New York: Springer, 2010. P. 561-584.

3. ABEND, Gabriel. Thick Concepts and the Moral Brain. In: European Journal of Sociology 52(1), 2011, p. 143-72.

4. ABEND, Gabriel. What the Science of Morality Doesn't Say About Morality. Philosophy of the Social Sciences, 2012, p. 1-42.

5. ABEND, Gabriel. The Moral Background: An Inquiry into the History of Business Ethics. Princeton, New Jersey: Princeton University Press, 2014.

6. ANTONACCIO, Olena; TITTLE, Charles R.. Morality, Self-Control, and Crime. In: Criminology, 46(2), 2008, p. 479-510.

7. AQUINO, Karl; REED, Americus. The Self-Importance of Moral Identity. In: Journal of Personality and Social Psychology, 83(6), 2002, p. 1423-1440.

8. BADER, Christopher D; FINKE, Roger. What does God require? Understanding religious context and morality. In: HITLIN, Steven; VAISEY, Stephen (ed.). Handbook of the Sociology of Morality. New York: Springer, 2010. P. 241-54

9. BANDURA, Albert. Moral Disengagement in the Perpetration of Inhumanities. In: Personality and Social Psychology Review, 3(3), 1999, p. 193-209.

10. BANDURA, Albert. Selective Exercise of Moral Agency. In: THORKILDSEN, T. A.; WALBERG, H.J. (eds.). Nuturing Morality. Boston: Kluwer Academic, 2004. P. 37-57.

11. BANDURA, Albert. Toward an Agentic Theory of Self. In: MARSH, Herbert W.; CRAVEN, Rhonda G.; MCINERNEY, Dennis M. (eds.). Self-Processes, Learning, and Enabling Human Potential. United States: Information Age Publishing, 2008. P. 15-49.

12. BANDURA, Albert; BARBANELLI, Claudio; CAPRARA, Gian Vittorio; PASTORELLI, Concetta. Mechanisms of Moral Disengagement in the Exercise of Moral Agency. In: Journal of Personality and Social Psychology, 71(2), 1996, p. 364-374. 
13. BLACK, Donald. Moral Time. New York: Oxford University Press, 2011.

14. BLAIR-LOY, Mary. Competing Devotions: Career and Family among Women Executives. Cambirdge, MA: Harvard University Press, 2003.

15. BLAIR-LOY, Mary. Moral Dimensions of the Work-Family Nexus. In: HITLIN, Steven; VAISEY, Stephen (ed.). Handbook of the Sociology of Morality. New York: Springer, 2010. P. 439-53.

16. BLASI, Augusto. Moral Functioning: Moral Understanding and Personality. In: LAPSLEY, Daniel K.; NARVAEZ, Darcia (eds.). Moral Development, Self, and Identity. Mahwah, N.J.: Lawrence Erlbaum Associates, 2004. P. 335-48.

17. CALHOUN, Craig. Morality, Identity, and Historical Explanation: Charles Taylor on the Sources of the Self. In: Sociological Theory 9(2), 1991, p. 232-263.

18. COLLINS, Randall. On the microfoundations of macrosociology. In: American Journal of Sociology, 1981, p. 984-1014.

19. DAMASIO, Antonio. The Feeling of What Happens. San Diego: Harcourt, 1999.

20. DAMASIO, Antonio. Looking For Spinoza: Joy, Sorrow, and the Feeling Brain. Orlando: Harcourt, 2003.

21. DAMASIO, Antonio. The Neurobiological Grounding of Human Values. In: CHANGEUX, Jean-Pierre; SINGER, Wolf; DAMASIO, Antonio; CHRISTEN, Yves (eds.). Neurobiology of Human Values. Germany: Springer-Verlag, 2005. P. 4756.

22. DE WAAL, Frans. Primates and Philosophers: How Morality Evolved. United States of America: Princeton University Press, 2006.

23. DE WAAL, Frans. The Age of Empathy: Nature's Lessons for a Kinder Society. New York: Crown Publishing Group, 2009.

24. DURKHEIM, Emile. The Elementary Forms of Religious Life. New York: Free Press, 1965[1912].

25. ELDER JR. Glen H.. Time, Human Agency, and Social Change: Perspectives on the Life Course. In: Social Psychology Quarterly 57(1), 1994, p. 4-15.

26. FIRAT, Rengin; HITLIN, Steven. Morally Bonded and Bounded: A Sociological Introduction to Neurology. In: Advances in group processes, 29, 2012 , p. 165-99.

27. FIRAT, Rengin; MCPHERSON, Chad Michael. Toward an Integrated Science of Morality. In: HITLIN, Steven; VAISEY, Stephen (ed.). Handbook of the Sociology of Morality. New York: Springer, 2010. P. 361-384. 
28. FISKE, Alan P.. The four elementary forms of sociality: framework for a unified theory of social relations. In: Psychological review, 99(4), 1992.

29. FISKE, Alan P.; HASLAM, Nick. The Four Basic Social Bonds: Structures for Coordinating Interaction. In: BALDWIN, Mark W. (ed.). Interpersonal Cognition. New York: Guilford Press, 2005. P. 267-298.

30. FLACK, Jessica C.; DE WAAL, Frans B.M.. Any Animal Whatever: Darwinian Building Blocks of Morality in Monkeys and Apes. In: Journal of Consciousness Studies 7(1-2), 2000, p. 1-29.

31. FOURCADE, Marion; HEALY, Kieran. Moral Views of Market Society. In: Annual Review of Sociology, 33, 2007, p. 285-311.

32. FRANKS, David D.. Neurosociology: The Nexus between Neuroscience and Social Psychology. New York: Springer, 2010.

33. FREESE, Jeremy. Genetics and the Social Science Explanation of Individual Outcomes. In: American Journal of Sociology, 114(S1), 2008, S1-S35.

34. FRIMER, Jeremy A.; WALKER, Lawrence J.. Reconciling the Self and Morality: An Empirical Model of Moral Centrality Development. In: Developmental Psychology 45(6), 2008, p. 1669-1681.

35. GARFINKEL, Howard. Studies in Ethnomethodology. New Jersey: Prentice Hall, 1967.

36. GIDDENS, Anthony (Ed.). The constitution of society introduction of the theory of structuration. Berkeley: University of California Press, 1984.

37. GIGERENZER, Gerd. Moral Satisficing: Rethinking Moral Behavior as Bounded Rationality. In: Topics in Cognitive Science, 2, 2010, 528-554.

38. GILLIGAN, Carol (Ed.). In a different voice psychological theory and women's development. Cambridge, Mass: Harvard University Press, 1982.

39. GOFFMAN, Erving (Ed.). The presentation of self in everyday life. Garden City, N.Y: Doubleday, 1959.

40. GOFFMAN, Erving. The Interaction Order: American Sociological Association, 1982 Presidential Address. In: American Sociological Review 48(1), 1983, p. 1-17.

41. GRAHAM, Jesse; HAIDT, Jonathan; KOLEVA, Spassena; MOTYL, Matt; IYER, Ravi; WOJCIK, Sean; DITTO, Peter. Moral foundations theory: The pragmatic validity of moral pluralism. Advances in Experimental Social Psychology, Forthcoming. 2012.

42. GRAHAM, Jesse; NOSEK, Brian A.; HAIDT, Jonathan; IYER, Ravi; KOLEVA, Spassena; DITTO, Peter H.. Mapping the Moral Domain. In: Journal of Personality and Social Psychology On-Line Early, 2011. 
43. GREENE, Joshua. Moral Tribes: Emotion, Reason, and the Gap Between Us and Them. New York: Penguin, 2013.

44. GREENE, Joshua; HAIDT, Jonathan. How (and Where) Does Moral Judgement Work? In: Trends in Cognitive Sciences, 6(12), 2002, p. 517-523.

45. HAIDT, Jonathan. The Emotional Dog and Its Rational Tail: A Social Intuitionist Approach to Moral Judgement. In: Psychological review 108(4), 2001, p. 814-34.

46. HAIDT, Jonathan. The Moral Emotions. In: DAVIDSON, R.J.; SCHERER, K.R.; GOLDSMITH, H.H. (Eds.). Handbook of Affective Sciences. Oxford: Oxford University Press, 2003. P. 852-870.

47. HAIDT, Jonathan; HERSH, Matthew A.. Sexual Morality: The Cultures and Emotions of Conservatives and Liberals. In: Journal of Applied Social Psychology, 31(1), 2001, p. 191-221.

48. HAIDT, Jonathan; JOSEPH, Craig. The Moral Mind: How Five Sets of Innate Intuitions Guide the Development of Many Culture-Specific Virtues, and Perhaps Even Modules. In: CARRUTHERS, P.; LAURENCE, S.; STITCH, S. (Eds.). The Innate Mind. New York: Oxford Press, 2008. P. 367-92.

49. HAIDT, Jonathan; KESEBIR, Selin. Morality. In: FISKE, Susan; GILBERT, Daniel; LINDZEY, Gardner (Eds.). Handbook of Social Psychology. Hoboken, NJ: Wiley, 2010. P. 797-832.

50. HAIDT, Jonathan, KOLLER, Silvia Helena; DIAS, Maria G.. Affect, Culture, and Morality, or Is It Wrong to Eat Your Dog? In: Journal of Personality and Social Psychology 65(4), 1993, p. 613-28.

51. HALTEMAN, James; NOELL, Edd. Reckoning with Markets: Moral Reflection in Economics. Oxford: Oxford University Press, 2012.

52. HART, Daniel; CARLO, Gustavo. Moral Development in Adolescence. In: Journal of Research on Adolescence 15(3), 2005, p. 223-233.

53. HEALY, Kieran. Last Best Gifts: Altruism and the Market for Human Blood and Organs. Chicago: University of Chicago Press, 2006.

54. HEGTVEDT, Karen A.; SCHEUERMAN, Heather L.. 2010. The Justice/Morality Link: Implied, then Ignored, yet Inevitable. In: HITLIN, Steven; VAISEY, Stephen (eds.). Handbook of the Sociology of Morality. New York: Springer, 2010. P. 331-360.

55. HENRICH, Joseph; HEINE, Steven J.; NORENZAYAN, Ara. Most people are not WEIRD. In: Nature 466(7302), 2010, p. 29-29.

56. HENRICH, Joseph HEINE, Steven J.; NORENZAYAN, Ara. The Weirdest People in the World? In: Behavioral and Brain Sciences 33(2/3), 2010, p. 1-75. 
Sociologias, Porto Alegre, ano 17, no 39, mai/ago 2015, p. 26-58

57. HITLIN, Steven. Moral Selves, Evil Selves: The Social Psychology of Conscience. New York: Palgrave Macmillan, 2008.

58. HITLIN, Steven; ANDERSSON, Matthew A.. Dignity. In: BRUNSMA, David L.; SMITH, Keri E. lyall; BRAN, Brian K. (eds.). Handbook of Sociology and Human Rights. Boulder, CO: Paradigm, 2013. P. 384-393.

59. HITLIN, Steven; VAISEY, Stephen (Eds.). Handbook of the Sociology of Morality. New York: Springer, 2010.

60. HITLIN, Steven; VAISEY, Stephen. The New Sociology of Morality. In: Annual Review of Sociology 39, 2013, p. 51-68.

61. HOFFMAN, Martin L.. Empathy and Moral Development: Implications for Caring and Justice. United States of America: Cambridge, 2000.

62. IGNATOW, Gabriel. Culture and Embodied Cognition: Moral Discourses in Internet Support Groups for Overeaters. In: Social Forces 88(2), 2009a, p. 643669.

63. IGNATOW, Gabriel. Why the Sociology of Morality Needs Bourdieu's Habitus. In: Sociological Inquiry 79(1), 2009b, p. 98-114.

64. INGLEHART, Ronald; BAKER, Wayne E.. Modernization, Cultural Change, and the Persistance of Traditional Values. In: American Sociological Review, 65(1), 2000, p. 19-51.

65. JACKALL, Robert. Moral Mazes: The World of Corporate Managers. New York: Oxford University Press, 1988.

66. JAFFEE, Sara; HYDE, Janet Shibley. Gender Differences in Moral Orientation: A Meta-Analysis. In: Psychological Bulletin, 126(5), 2000, p. 703-726.

67. JAHODA, Gustav. A History of Social Psychology: From the Eighteenth-Century Enlightenment to the Second World War. United Kingdom: Cambridge, 2007.

68. JOAS, Hans (Ed.). The Genesis of Values. Cambridge, UK: Polity Press, 2000.

69. JOAS, Hans; KNOBL, Wolfgang. Social Theory: Twenty Introductory Lectures. Cambridge: Cambridge University Press, 2009.

70. KAGAN, Jerome. The Nature of the Child. United States of America: HarperCollins, 1994[1984].

71. KATZ, Jack. Essences as Moral Identities: Verifiability and Responsibility in Imputations of Deviance and Charisma. In: American Journal of Sociology, 80(6), 1975, p. 1369-1390.

72. KOCHANSKA, Grazyna. Committed Compliance, Moral Self, and Internalization: A Mediational Model. In: Developmental Psychology, 38(3), 2002, p.339-51. 
73. KOCHANSKA, Grazyna; AKSAN, Nazan; KNAACK, Amy; RHINES, Heather M.. Maternal Parenting and Children's Conscience: Early Security as Moderator. In: Child development 75(4), 2004, p. 1229-1242.

74. KOHLBERG, Lawrence. The Philosophy of Moral Development. Vol I - Moral Stges and the Idea of Justice. New York: Harper \& Row, 1981.

75. KREBS, Dennis L. The Origins of Morality: An Evolutionary Account. New York: Oxford University Press, 2011.

76. LAMONT, Michele. Money, Morals, and Manners: The Culture of the French and the American Upper-Middle Class. United States of America: University of Chicago Press, 1992.

77. LAPSLEY, Daniel K.; NARVAEZ, Darcia. Moral Development, Self, and Identity. Mahwah, N.J.: Lawrence Erlbaum Associates, 2004.

78. LEVINE, Donald N. Adumbrations of a Sociology of Morality in the Work of Parsons, Simmel, and Merton. In: HITLIN, Steven; VAISEY, Stephen (eds.). Handbook of the Sociology of Morality. New York: Springer, 2010. P. 57-72.

79. LUKES, Steven. Emile Durkheim: his life and work; a historical and critical study. Stanford University Press, 1985.

80. LUKES, Steven. Moral Relativism. New York: Picador, 2008.

81. LUKES, Steven. 2010. The Social Construction of Morality? In: HITLIN, Steven; VAISEY, Stephen (eds.). Handbook of the Sociology of Morality. New York: Springer, 2010. P. 549-560.

82. MARYANSKI, Alexandra; TURNER, Jonathan H.. The Social Cage: Human Nature and the Evolution of Society. Stanford, CA: Stanford University Press, 1992.

83. MASSEY, Douglas S.. A Brief History of Human Society: The Origin and Role of Emotion in Social Life. In: American Sociological Review 67(1), 2002, p. 1-29.

84. MEAD, George Herbert (Ed.). The Philosophy of the Present. Chicago, London: Open Court Publishing, 1932.

85. MEAD, George Herbert (Ed.). Mind, Self \& Society from the Standpoint of a Social Behaviorist. Chicago, Ill: The University of Chicago press, 1934.

86. NUNNER-WINKLER, Gertrud. The Development of Moral Understanding and Moral Motivation. In: International Journal of Educational Research 27(7), 1998, p. 587-603.

87. OSOFSKY, Michael J.; BANDURA, Albert; ZIMBARDO, Philip G.. The Role of Moral Disengagement in the Execution Process. In: Law and Human Behavior, 29(4), 2005, p. 371-393. 
88. PILIAVIN, Jane Allyn. Altruism and Helping: The Evolution of a Field: The 2008 Cooley-Mead Presentation. In: Social Psychology Quarterly 72(3), 2008, p. 209-225.

89. POWELL, Christopher. Four Concepts of Morality. In: HITLIN, Steven; VAISEY, Stephen (eds.). Handbook of the Sociology of Morality. New York: Springer, 2010. P. 35-56.

90. PRINZ, Jesse J.. The Emotional Construction of Morals. New York: Oxford, 2007.

91. RAI, Tage Shakti; FISKE, Alan Page. Moral psychology is relationship regulation: moral motives for unity, hierarchy, equality, and proportionality. In: Psychological review 118(1):57, 2011.

92. RAWLS, Anne Warfield. The Interaction Order Sui Generis:Goffman's Contribution to Social Theory. In: Sociological Theory, 5(2), 1987, p. 136-149.

93. RAWLS, Anne Warfield. Language, Self, and Social Order: A Reformulation of Goffman and Sacks. In: Human Studies, 12(1-2), 1989, p. 147-172.

94. RAWLS, Anne Warfield. Social Order as Moral Order. In: HITLIN, Steven; VAISEY, Stephen (eds.). Handbook of the Sociology of Morality. New York: Springer, 2010. P. 95-122.

95. REED, Americus; AQUINO, Karl F.. Moral Identity and the Expanding Circle of Regard Toward Out-Groups. In: Journal of Personality and Social Psychology, 84(6), 2003, p. 1270-1286.

96. ROZIN, Paul; LWERY, LaurA; IMADA, Sumio; HAIDT, Jonathan. The CAD Triad Hypothesis: A Mapping Between Three Moral Emotions (Contempt, Anger, Disgust) and Three Moral Codes (Community, Autonomy, Divinity). In: Journal of Personality and Social Psychology, 76(4), 1999, p. 574-586.

97. SAYER, Andrew. The Moral Significance of Class. New York: Cambridge University Press, 2005.

98. SAYER, Andrew. Why Things Matter to People: Social Science, Values, and Ethical Life. Cambridge: Cambridge University Press, 2011.

99. SCHEFF, Thomas J.. Shame and the Social Bond: A Sociological Theory. In: Sociological Theory, 18(1), 2000, p. 84-99.

100. SCHEFF, Thomas J.. Shame in Self and Society. In: Symbolic Interaction, 26(2), 2003, p. 239-62.

101. SCHWALBE, Michael L. Social Structure and the Moral Self. Pp. 281-303 In: HOWARD, Judith A.; CALLERO, Peter L. (eds.). The Self-Society Dynamic: Cognition, Emotion, and Action. New York: Cambridge, 1991. 
102. SCHWARTZ, Shalom. Toward Refining the Theory of Basic Human Values. In: SALZBORN, Samuel; DAVIDOV, Eldad; REINECKE, Jost (eds.). Methods, Theories, and Empirical Applications in the Social Sciences. VS Verlag für Sozialwissenschaften, 2012. P. 39-46.

103. SCOTT, James C.. The Moral Economy of the Peasant. New Haven: Yale University Press, 1976.

104. SHWEDER, Richard A.; HAIDT, Jonathan. The Future of Moral Psychology: Truth, Intuition, and the Pluralist Way. In: Psychological Science, 4(6), 1993, p. 360-365.

105. SHWEDER, Richard A.; MAHAPATRA, Manamohan; MILLER, Joan. Culture and Moral Development. In: KAGAN, Jerome; LAMB, Sharon (eds). The Emergence of Morality in Young Children. Chicago: University of Chicago Press, 1987. P. 1-83.

106. SHWEDER, Richard A.; MAHAPATRA, Manamohan; MILLER, Joan. Culture and Moral Development. In: SIGLER, James W.; SHWEDER, Richard A; HERDT, Gilbert (eds.). Cultural Psychology: Essays on Comparative Human Development. Cambridge: Cambridge University Press, 1990. P. 130-204.

107. SINNOTT-ARMSTRONG, Walter. Moral Psychology: The cognitive science of morality: intuition and diversity. The MIT Press, 2008.

108. SKITKA, Linda J.; BAUMAN, Christopher W.; MULLEN, Elizabeth. Morality and Justice: An Expanded Theoretical Perspective and Empirical Review. In: Advances in group processes, 25, 2008, p. 1-27.

109. SMITH, Christian. Moral, Believing Animals: Human Personhood and Culture. United States of America: Oxford University Press, 2003.

110. SMITH, Christian. What is a Person? Chicago: University of Chicago Press, 2009.

111. SNYDER, Benjamin H. From Vigilance to Busyness: A Neo-Weberian Approach to Clock Time. In: Sociological Theory, 31(3), 2013, p. 243-266.

112. SPATES, James L. The Sociology of Values. In: Annual Review of Sociology, 9, 1983, p. 27-49.

113. STARKS, Brian; ROBINSON, Robert V.. Moral Cosmology, Relgion, and Adult Values for Children. In: Journal for the Scientific Study of Religion, 46(1), 2007, p. 17-35.

114. STAUB, Ervin. The Psychology of Good and Evil: Why Children, Adults, and Groups Help and Harm Others. United States: Cambridge, 2003.

115. STEENSLAND, Brian. Moral classification and social policy. In: HITLIN, Steven; VAISEY, Stephen (ed.). Handbook of the Sociology of Morality. New York: Springer, 2010. P. 455-468. 
116. STETS, Jan E.; CARTER, Michael J.. The Moral Identity: A Principle Level Identity. P. 293-316 In: MCCLELLAND, Kent; FARARO, Thomas (eds.). Purpose, Meaning, and Action: Control System Theories in Sociology. New York: Palgrave Macmillan, 2006.

117. STETS, Jan E.; CARTER, Michael J.. A Theory of the Self for the Sociology of Morality. In: American Sociological Review, 77(1), 2012, P. 120-140.

118. SUNAR, Diane. Suggestions for a New Integration in the Psychology of Morality. In: Social and Personality Psychology Compass, 3(4), 2009, p. 447-474.

119. TANGNEY, June Price; STUEWIG, Jeff; MASHEK, Debra J.. Moral Emotions and Moral Behavior. In: Annual review of psychology, 58, 2007, p. 345-372.

120. TAVORY, Iddo. The Question of Moral Action: A Formalist Position. In: Sociological Theory 29(4), 2011, p. 272-293.

121. TAYLOR, Charles (Ed.). Sources of the Self: The Making of the Modern Identity. Cambridge, Mass: Harvard University Press, 1989.

122. TURIEL, Elliot. The Development of Social Knowledge: Moralty and Convention. Cambridge: Cambridge University Press, 1983.

123. TURIEL, Elliot.. The Culture of Morality: Social Development, Context, and Conflict. New York: Cambridge University Press, 2002.

124. TURIEL, Elliot; KILLEN, M.; HELWIG, Charles C.. Morality: Its Structure, Functions and Vagarities. P. 155-243 In: KAGAN, Jerome; LAMB, S. (eds.). The Emergence of Morality in Young Children. Chicago: University of Chicago Press, 1987.

125. TURNER, Jonathan H; STETS, Jan E.. Moral emotions. In: TURNER, Jonathan H; STETS, Jan E.. (eds.). Handbook of the Sociology of Emotions. Springer, 2006a. P. 544-566.

126. TURNER, Jonathan H. Natural Selection and the Evolution of Morality in Human Societies. In: HITLIN, Steven; VAISEY, Stephen (ed.). Handbook of the Sociology of Morality. New York: Springer, 2010. P. 125-145.

127. TURNER, Jonathan H; STETS, Jan E.. Sociological Theories of Human Emotions. In: Annual Review of Sociology, 32, 2006b, p. 25-52.

128. TURNER, Ralph H. Articulating Self and Social Structure. In: YARDLEY, K.; HONESS, T. (eds.). Self and Identity: Psychosocial Perspectives. United States of America: John Wiley \& Sons, Ltd, 1987. P. 119-132.

129. USLANER, Eric. The Moral Foundations of Trust. United Kingdom: Cambridge, 2002.

130. VAISEY, Stephen. Structure, Culture, and Community: The Search for Belonging in 50 Urban Communes. In: American Sociological Review, 72(6), 2007, p. 851-873. 
131. VAISEY, Stephen. Motivation and Justification: A Dual-Process Model of Culture in Action. In: American Journal of Sociology, 114(6), 2009, p. 1675-1715.

132. VAISEY, Stephen; LIZARDO, Omar. 2010. Can Cultural Worldviews Influence Network Composition? In: Social Forces, 88(4), 2010, p. 1595-1618.

133. WALKER, Janet S. Choosing Biases, Using Power, and Practicing Resistance: Moral Development in a World Without Certainty. In: Human Development, 43, 2000, p. 135-156.

134. WIKSTROM, Per-Lofo H.; CECCATO, Vania; HARDIE, Beth; TREIBER, Kyle. Activity Fields and the Dynamics of Crime: Advancing Knowledge About the Role of the Environment in Crime Causation. In: Journal of Quantitative Criminology $26: 1,2010$.

135. WIKSTROM, Per-Lofo H.. Deterrence and Deterrence Experiences: Preventing Crime through the Threat of Punishment. In: SHOHAM, Shlomo Giora; BECK, Ori; KETT, Martin (eds.). International Handbook of Penology and Criminal Justice. London: CRC Press, 2007. P. 345-78.

136. WINCHESTER, Daniel. Embodying the Faith: Religious Practice and the Making of a Muslim Moral Habitus. In: Social Forces, 86(4), 2008.

137. WUTHNOW, Robert. Meaning and Moral Order. Berkeley: University of California Press, 1992.

138. ZELIZER, Viviana A.. The purchase of intimacy. Princeton University Press, 2007.

139. ZELIZER, Viviana A.. Morals \& Markets: The Development of Life Insurance in the United States. New Brunswick: Transaction Books, 1979.

Recebido em: 10/09/2014.

Aceite Final: 14/10/2014. 